\title{
Comparison of turbulence models for the computational fluid dynamics simulation of wind turbine wakes in the atmospheric boundary layer
}

\author{
D. Cabezón E. Migoya and A. Crespo
}

\begin{abstract}
An elliptic computational fluid dynamics wake model based on the actuator disk concept is used to simulate a wind turbine, approximated by a disk upon which a distribution of forces, defined as axial momentum sources, is applied on an incoming non-uniform shear flow. The rotor is supposed to be uniformly loaded with the exerted forces estimated as a function of the incident wind speed, thrust coefficient and rotor diameter. The model is assessed in terms of wind speed deficit and added turbulence intensity for different turbulence models and is validated from experimental measurements of the Sexbierum wind turbine experiment.

The elliptic model is also compared with other wake models in the literature on the basis of parabolic and large eddy simulation approximations. Copyright (C) 2011 John Wiley \& Sons, Ltd.
\end{abstract}

\section{KEYWORDS}

actuator disk; thrust coefficient; turbulence modeling

\section{INTRODUCTION}

Wind turbine wake effect has been modeled using different approaches during the last years, ${ }^{1-3}$ from analytical engineering models to three-dimensional computational fluid dynamics (CFD) full rotor modeling. Nevertheless, because of the need for modeling wind farm aerodynamics in challenging new environments such as complex terrain and offshore, several proposals have come out through the so-called back to basics concept. That is why more sophisticated wake models are needed for the estimation of power deficits in these types of environments where new effects such as wake-terrain interaction as well as stability effects are known to have a significant impact on wake evolution and power losses. Despite that, these new models need to be first assessed and calibrated in flat terrain where existing homogeneous and neutral conditions allow better calibration procedures.

Nevertheless, the main disadvantage from the modeling point of view consists in the existence of important differences in the size of turbulent length scales between the atmospheric boundary layer (ABL) and wake areas inside wind farms, which poses a serious problem for modelers. This brings as a consequence that traditional turbulence models used for the simulation of the ABL wind flow cannot be applied directly to wind farm analysis, so some modifications have to be made to them.

On the one hand, second-order closure turbulence models such as the standard $k-\varepsilon$ turbulence model based on the eddy viscosity concept are quite cheap in terms of computational effort but usually fail at estimating turbulent viscosity inside the wake area because of the excessive modeled turbulent diffusion. In order to mitigate this, some modifications, leading to some improvements, have been proposed over the standard form of the $k-\varepsilon$ turbulence model. ${ }^{4-7}$ 
On the other hand and together with the previous corrections, another anisotropic turbulence model based on the solution of the Reynolds stress tensor may represent an interesting alternative to second-order closures in order to face the length scale imbalance although this implies an increase in computational time.

Among the different alternatives for modeling wind turbine rotors, an elliptical approach based on coupling the actuator disk technique and numerical CFD modeling, representing an acceptable approximation of the rotor without leaving the main essence of the involved physics, is presented. The model is based on the thrust-velocity curve (so the geometry of the rotor is not considered) and calculates the extraction of axial momentum from the incoming flow at the rotor face by applying a uniform distribution of forces. Using these simplified techniques instead of CFD-BEM (Blade Element-momentum theory) coupling have been demonstrated to have negligible impact on the axial evolution of the far wake, ${ }^{7}$ where interference between wakes, turbulence modeling and topographical effects are usually more relevant at estimating power losses because of wake effect. ${ }^{1}$

This analysis looks for possible solutions to model simultaneously rotor wake aerodynamics and surface boundary layer (SBL) meteorology as its bounding environment. For that purpose, different turbulence models, going from its standard $k-\varepsilon$ form $^{8}$ up to the realizable $k-\varepsilon^{9}$ as well as an additional approach through the anisotropic model Reynolds stress model (RSM), ${ }^{10}$ are assessed. The results obtained from the actuator disk technique are compared with other wake models such as the parabolic UPMPARK mode $1^{10}$ and are validated using the field data from the Sexbierum experiment ${ }^{11}$ in order to observe the accuracy on the prediction of wind speed deficit and added turbulence intensity inside wake flow. Although parabolic models are less accurate than elliptic models, they consume less computing time, and the comparison between results of both models may be useful for engineering applications. Nevertheless, the focus of this paper is on the comparison of elliptic models with experiments, and the comparison with other models is mainly presented to quantify the improvement of the results.

These results represent the first stage for future developments on CFD wake modeling, in particular, on all those related to meshing strategies and turbulence modeling in order to simulate rotor wakes for large complex terrain and offshore wind farms in a more efficient way.

\section{SEXBIERUM EXPERIMENT}

The main objective of the Sexbierum experiment consisted of measuring and analyzing the wake flow downwind of a three-bladed $300 \mathrm{~kW}$ wind turbine, with a rotor diameter of $30 \mathrm{~m}$ and a hub height of $35 \mathrm{~m} .{ }^{11}$ For that purpose, a meteorological mast was located $2.8 \mathrm{D}$ upstream of the wind turbine and another three met masts downstream, at distances of $2.5 \mathrm{D}$, $5.5 D$ and $8 D$, in order to characterize the near and far wakes (Figure 1). For the measurement period, the upstream mast registered at hub height a free stream velocity of $10 \mathrm{~m} \mathrm{~s}^{-1}$ and a turbulence intensity of $10 \%$, equivalent to an axial thrust coefficient of $C_{\mathrm{t}}=0.75$ and a local roughness length of $z_{0}=0.0018 \mathrm{~m}$ under neutral stability conditions, respectively. These parameters are used for the definition of the incoming flow at the inlet of the domain for the rotor simulation.

\section{NUMERICAL MODEL}

\subsection{Surface Boundary Layer modeling}

The free stream flow upstream of the wind turbine corresponds to the fully developed vertical profiles in the surface boundary layer (SBL), characterized by a non-uniform shear boundary layer flow. The CFD code CFDWind $1.0^{12}$ based on the commercial software package FLUENT 6.3 (ANSYS, Inc., Canonsburg, PA) is adopted in order to solve the mean wind speed components and turbulence intensity according to the Monin-Obukhov theory. The expressions that describe this flow are derived from Panofsky and Dutton. ${ }^{13}$

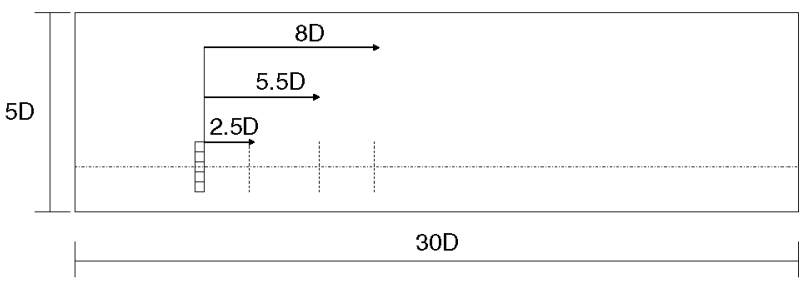

Figure 1. Location of met masts at the positions downstream of the rotor. 
Turbulent viscosity is assumed to vary linearly with height:

$$
\mu_{\mathrm{t}}(z)=\kappa u * z
$$

where $u_{*}$ is the friction velocity $\left(\mathrm{m} \mathrm{s}^{-1}\right), \kappa=0.4$ is the von Karman constant and $z$ is the height above the ground ( $\mathrm{m}$ ).

Neutral atmosphere is supposed, so thermal effects, together with the Coriolis effects, are neglected. Assuming shear stress to be constant over the SBL, a logarithmic velocity profile is defined at the entrance of the domain as

$$
u(z)=\frac{u_{*}}{\kappa} \ln \left(\frac{z}{z_{0}}\right)
$$

where $z_{0}$ is the equivalent roughness length $(\mathrm{m})$.

Assuming equilibrium between the production and the dissipation rates of the turbulent kinetic energy, the vertical profiles for the turbulent quantities, which turns out to be constant with height, can be derived as

$$
k(z)=\frac{u_{*}^{2}}{\sqrt{C_{\mu}}}
$$

for the turbulent kinetic energy, where $C_{\mu}$ is a constant of the standard $k-\varepsilon$ turbulence model. On the other hand,

$$
\varepsilon(z)=\frac{u_{*}^{3}}{\kappa z}
$$

for the dissipation rate of the turbulent kinetic energy.

\subsection{Actuator disk model}

From the linear momentum theory, in its simplest form, it can be derived that the axial force from which the wind turbine extracts the kinetic energy from the incoming air flow is just a function of the local induction factor, or alternatively, of the thrust coefficient for the corresponding upstream wind speed. $4,5,14$ The wind turbine is thus considered as an actuator disk upon which a uniform distribution of axial forces, defined as momentum sink terms, is applied. These local axial forces can be prescribed over the disk area as

$$
F=0.5 \cdot \rho \cdot \frac{\pi D^{2}}{4} \cdot C_{t} \cdot V_{\mathrm{inf}}^{2}
$$

where $D$ is the rotor diameter $\left(\mathrm{m}^{2}\right) ; V_{\text {inf }}$ is the upstream wind speed $\left(\mathrm{m} \mathrm{s}^{-1}\right)$, obtained, in this case, from the upstream mast; $C_{\mathrm{t}}$ is the thrust coefficient, obtained from the $C_{\mathrm{t}}$-velocity curve for the corresponding value of $\mathrm{V}_{\text {inf }}$; and $\rho$ is the air density $\left(\mathrm{kg} \mathrm{m}^{-3}\right)$.

The local prescribed force is to be applied on a volume of cells defining the rotor in units of force per cubic meter $\left(\mathrm{N} / \mathrm{m}^{3}\right)$.

\subsection{Numerical method}

The model is solved in a computational domain of $30 D$ in the axial direction $(X), 7 D$ in the cross direction $(Y)$ and $5 D$ in the vertical direction $(Z)$. For that purpose, a structured grid was generated with the commercial software package ICEM CFD 11.0 HEXA (ANSYS, Inc.), getting a computational domain of approximately 230,000 hexas, solved by the commercial CFD solver FLUENT 6.3.

The choice of the top height has been based on the wind engineering reference guideline of $5 H$ ( $H$, height of any obstacle) to avoid flow blockage. The prescribed boundary conditions correspond to the SBL modeling, where the Monin-Obukhov theory is valid up to the first $100 \mathrm{~m}$. Beyond this height, the results are no longer representative, so the height of the top boundary should be placed such that the flow is not blocked. Calculations show that the wind speed deficit remains the same for higher values of the top height.

\subsubsection{Grid independence analysis}

From the meshing point of view, there are no previous guidelines on grid resolution at the rotor position for this actuator-disk-type modeling. That is why a grid independence study has to be made in order to estimate the optimum number of cells to be used.

Different resolutions are tested in order to observe the evolution of the wind speed deficit modeled by the actuator disk in the axial, cross and vertical directions. 
The default vertical distribution of nodes corresponds to the mostly recommended one in the definition of the SBL with a variable cell size, a height of the first cell centroid close to the ground set to $0.5 \mathrm{~m}$ and a geometrically growing ratio of 1.12, getting around 15 nodes below hub height, in order to capture the main velocity gradients near the ground.

Table I together with Figure 2 shows the variability on the wind speed ratio for different grid spacings on the axial direction corresponding to the thickness of the rotor disk. The results are prescribed at the near wake section located at $2.5 \mathrm{D}$ downstream of the rotor disk where grid independence is reached after the far wake sections.

As shown, grid independence analysis is reached for a rotor thickness of $0.06 \mathrm{D}$, with a negligible level of variability. The growing ratio of nodes behind the rotor disk, corresponding to a geometric factor of 1.04, is the same for all the cases.

A similar analysis is made for the cross direction, where the grid independence is reached for $0.16 D$, as observed in Table II for three different spacings.

Finally, a sensitivity analysis on the number of nodes below hub height is made, conditioning also the number of nodes to be included at the rotor disk (Table III). Slight differences observed from 15 nodes below hub height makes the grid independent at this stage.

The observed results lead to the conclusion that, for this case, a resolution of $0.06 \mathrm{D}$ in the axial direction, $0.16 \mathrm{D}$ in the cross direction and 20 nodes below hub height makes the results independent of the generated grid at all the sections, concluding, for this case, on an optimum rotor disk composed of 43 control volumes.

Table I. Grid independence analysis for axial direction. Results at 2.5D section ( $N_{z}$, number of nodes below hub height).

\begin{tabular}{lcccc}
\hline$\Delta \mathrm{X}$ & $\Delta \mathrm{y}$ & $\mathrm{Nz}$ & WS ratio & Variability (\%) \\
\hline $0.02 D$ & $0.16 D$ & 15 & 0.845 & 0.376 \\
$\mathbf{0 . 0 6 D}$ & & & $\mathbf{0 . 8 4 8}$ & 0.372 \\
$0.10 D$ & & & 0.845 & 0.907 \\
$0.16 D$ & & & 0.837 & 0.735 \\
$0.30 D$ & & & 0.831 & 1.066 \\
$0.60 D$ & & & 0.822 & - \\
\hline
\end{tabular}

WS, wind speed.

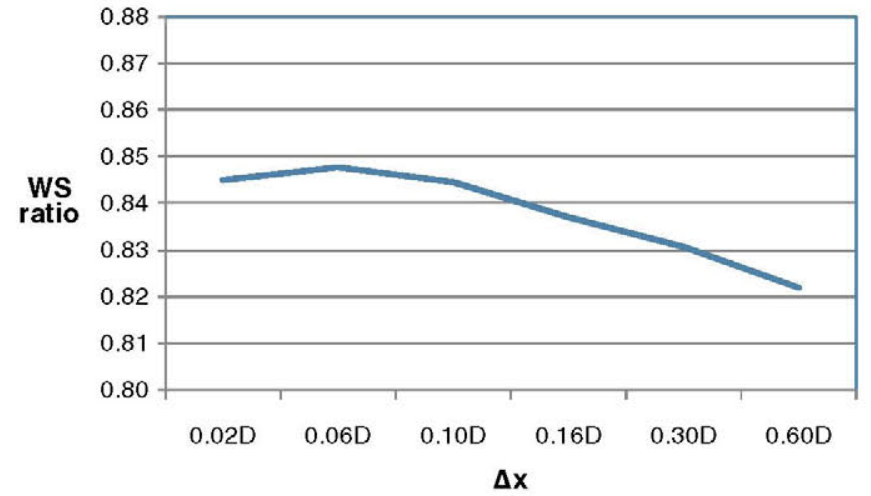

Figure 2. Evolution of wind speed (WS) ratio at $2.5 D$ section for different axial grid spacings.

Table II. Grid independence analysis for cross direction. Results at $2.5 D$ section ( $N_{z}$, number of nodes below hub height).

\begin{tabular}{lcccc}
\hline$\Delta X$ & $\Delta y$ & $N_{z}$ & WS ratio & Variability (\%) \\
\hline $0.06 D$ & $0.06 D$ & 15 & 0.848 & 0.000 \\
& $\mathbf{0 . 1 6 D}$ & & $\mathbf{0 . 8 4 8}$ & 0.005 \\
& $0.3 D$ & & 0.844 & - \\
\hline
\end{tabular}

WS, wind speed. 
Table III. Grid independence analysis for vertical direction. Results at 2.50 section ( $N_{z}$, number of nodes below hub height)

\begin{tabular}{lcccc}
\hline$\Delta \mathrm{X}$ & $\Delta \mathrm{y}$ & $N_{z}$ & WS ratio & Variability (\%) \\
\hline $0.06 D$ & $0.16 D$ & 25 & 0.844 & 0.000 \\
& & $\mathbf{2 0}$ & $\mathbf{0 . 8 4 4}$ & 0.431 \\
& 15 & 0.848 & 0.947 \\
& 10 & 0.856 & - \\
\hline
\end{tabular}

WS, wind speed.

\subsubsection{Boundary conditions}

The inlet boundary conditions of the domain are defined by the vertical profiles (2), (3) and (4) described on Section 3.3.1. The outlet boundary is defined as pressure outlet, the lateral boundary as zero gradient or symmetry, and the top boundary is defined by fixing the inlet values of wind speed, turbulent kinetic energy and turbulent dissipation rate at the top height and by assuming a constant friction velocity. Lateral boundaries are defined as zero gradient.

The ground is simulated as a wall, through an adaption of the standard wall functions, setting a link between the turbulent law of the wall modified for mechanical roughness and the SBL log law based on the roughness length. For that purpose, the method proposed by Blocken et al. ${ }^{15}$ for FLUENT 6.3 is used in order to ensure continuity, both for the functions and their first derivative:

$$
k_{\mathrm{s}}=\frac{E \cdot z_{0}}{c_{\mathrm{s}}}
$$

where $k_{\mathrm{s}}$ is the roughness height of wall function $(\mathrm{m}), c_{\mathrm{s}}$ is the roughness constant of wall function, $E=9.793$ is the log law of the wall constant and $z_{0}$ is the roughness length as defined in the ABL (m).

For a better convergence of the numerical scheme used in FLUENT 6.3, the height of the cell adjacent to the wall $z_{\mathrm{p}}$ should be equal or larger than $k_{\mathrm{s}}$. In this work, it has been imposed that $k_{\mathrm{s}}=z_{\mathrm{p}}$. From this condition and from equation (6), the value of $c_{\mathrm{S}}$ is obtained.

\subsubsection{Numerical discretization}

Once the grid is generated, the steady state three-dimensional Navier-Stokes equations are solved using the elliptic Reynolds-averaged Navier-Stokes (RANS) approximation. The set of equations is then composed of the continuity equation, the three momentum equations and the two transport equations for turbulence.

A control volume technique is used for converting the governing differential equations into algebraic equations that can be solved numerically. A second-order upwind scheme based on multilinear reconstruction approach ${ }^{16}$ is used for all dependent variables.

The computational time depends on the computed mesh, going from $2 \mathrm{~min}$ for the coarsest one to $30 \mathrm{~min}$ for the most refined one. For all of them, approximately 1000 iterations were needed using $1 \mathrm{e}-5$ as the convergence criteria for all the variables. The simulations were run in a workstation of 16 GB RAM, dual quad core Intel Xeon 2.5 GHz processor (Intel Corporation, Santa Clara, CA).

\section{TURBULENCE MODELING}

\subsection{Isotropic $k-\varepsilon$ standard}

This is the standard form of the $k-\varepsilon$ turbulence model proposed originally by Launder and Spalding ${ }^{8}$ and is widely used because of its robustness and economy when solving relatively simple flows. The turbulent kinetic energy $k$ and its dissipation rate $\varepsilon$ are obtained from the following transport equations:

$$
\begin{gathered}
\frac{\partial}{\partial t}(\rho k)+\frac{\partial}{\partial x_{i}}\left(\rho k u_{i}\right)=\frac{\partial}{\partial x_{i}}\left[\left(\mu+\frac{\mu_{\mathrm{t}}}{\sigma_{k}}\right) \frac{\partial k}{\partial x_{j}}\right]+G_{k}-\rho \varepsilon \\
\frac{\partial}{\partial t}(\rho \varepsilon)+\frac{\partial}{\partial x_{i}}\left(\rho \varepsilon u_{i}\right)=\frac{\partial}{\partial x_{j}}\left[\left(\mu+\frac{\mu_{\mathrm{t}}}{\sigma_{\varepsilon}}\right) \frac{\partial \varepsilon}{\partial x_{j}}\right]+C_{1 \varepsilon} \frac{\varepsilon}{k} G_{k}-C_{2 \varepsilon} \rho \frac{\varepsilon^{2}}{k}
\end{gathered}
$$

where $\mu_{\mathrm{t}}$ is the turbulent viscosity $\left(\mathrm{kg} \mathrm{m}^{-1} \mathrm{~s}^{-1}\right)$, computed as

$$
\mu_{\mathrm{t}}=\rho C_{\mu} \frac{k^{2}}{\varepsilon}
$$


$G_{k}$ is the generation of turbulent kinetic energy due to the mean velocity gradients $\left(\mathrm{kg} \mathrm{m}^{-1} \mathrm{~s}^{-3}\right)$, computed as

$$
G_{k}=\mu_{\mathrm{t}} S^{2}
$$

to be consistent with the Boussinesq hypothesis, where $S$ is the modulus of the mean rate-of-strain tensor, ${ }^{17}$ defined as

$$
S=\sqrt{2 S_{i j} S_{i j}}
$$

where $S_{i j}$ is

$$
S_{i j}=\frac{1}{2}\left(\frac{\partial u_{i}}{\partial x_{j}}+\frac{\partial u_{j}}{\partial x_{i}}\right)
$$

$C_{\mu}, C_{1 \varepsilon}, C_{2 \varepsilon}, \sigma_{k}, \sigma_{\varepsilon}$ are the set of model constants with the following default values:

$$
C_{\mu}=0.09, C_{1 \varepsilon}=1.44, C_{2 \varepsilon}=1.92, \sigma_{k}=1, \sigma_{\varepsilon}=1.3
$$

When applied to the resolution of the SBL, it is common practice to make use of the measured standard deviations recommended by Panofsky and Dutton, ${ }^{13}$ leading to the modified set of constants

$$
C_{\mu}=0.033, C_{1 \varepsilon}=1.176, C_{2 \varepsilon}=1.92, \sigma_{k}=1, \sigma_{\varepsilon}=1.3
$$

that meets the condition of local equilibrium at the wall according to Richards and Hoxey ${ }^{17}$ :

$$
\sigma_{\varepsilon}=\frac{\kappa^{2}}{\left(C_{2 \varepsilon}-C_{1 \varepsilon}\right) \sqrt{C_{\mu}}}
$$

The model is likely to be inaccurate at those cases where strong gradients exist such as areas of great curvature with recirculation, leading to an overestimation of the turbulent viscosity.

In this application, the flow is steady, and the first terms on the left-hand sides of equations (7) and (8) are zero.

\subsection{Isotropic $k-\varepsilon$ standard: correction in $\varepsilon$ equation}

A recent work by El Kasmi and Masson ${ }^{7}$ proposed a correction in the $\varepsilon$ equation by increasing locally the turbulent dissipation rate in a volume of $0.25 \mathrm{D}$ long adjacent to the rotor disk such that the excess of turbulent diffusion is mitigated.

This increase is defined by a source term in the $\varepsilon$ equation (8), allowing the improvement of energy transfer from the larger to the smaller scales of the turbulence. ${ }^{7}$ This source term is defined by

$$
S_{\varepsilon}=C_{\varepsilon 4} \frac{G_{k}^{2}}{\rho k}
$$

where $S_{\varepsilon}$ is the source term in the $\varepsilon$ equation $\left(\mathrm{m}^{2} \mathrm{~s}^{-3}\right), C_{\varepsilon 4}=0.37$ is the additional constant, $\rho$ is the air density $\left(\mathrm{kg} \mathrm{m}^{-3}\right)$ and $k$ is the turbulent kinetic energy $\left(\mathrm{m}^{2} \mathrm{~s}^{-2}\right)$.

Increasing locally the turbulent dissipation rate may lead to lower values of the length scale near the rotor disk, counteracting the imbalance between the wake flow and the free stream flow. The main shortcoming of this approach comes from the uncertainty on tuning the $C_{\varepsilon 4}$ constant value as well as the size of the non-equilibrium volume adjacent to the rotor disk. At this analysis, the default values proposed by El Kasmin and Masson ${ }^{7}$ are used.

\subsection{Isotropic $k-\varepsilon$ realizable}

The realizable model proposed by Shih et al. ${ }^{9}$ differs from the standard model in two different ways. First, a new turbulent viscosity is formulated involving a variable $C_{\mu}$ as a function of the mean strain rate and the turbulence fields in terms of $k$ and $\varepsilon$. This new value is computed in FLUENT $6.3^{18}$ from

$$
C_{\mu}=\frac{1}{A_{0}+A_{s} \frac{k \tilde{S}}{\varepsilon}}
$$

where $A_{0}=4.04$ is the constant of model; $\tilde{S}=\sqrt{S_{i j} S_{i j}}$, where the repeated index denotes summation; and $A_{s}$ is the parameter of the model defined as

$$
A_{s}=\sqrt{6} \cos \phi
$$


with

$$
\phi=\frac{1}{3} \frac{1}{\cos (\sqrt{6} W)}, \quad W=\frac{S_{i j} S_{j k} S_{k i}}{\tilde{S}^{3}}
$$

Second, a new transport equation for the dissipation rate $\varepsilon$ is proposed. In the same way, the constant associated with the generation of $\varepsilon$ is affected by the mean strain tensor, making the upper value to be limited according to equation (21).

$$
\frac{\partial}{\partial x_{j}}\left(\rho \varepsilon u_{j}\right)=\frac{\partial}{\partial x_{j}}\left[\left(\mu+\frac{\mu_{t}}{\sigma_{\varepsilon}}\right) \frac{\partial \varepsilon}{\partial x_{j}}\right]+\rho C_{1 \varepsilon} S \varepsilon-\rho C_{2 \varepsilon} \frac{\varepsilon^{2}}{k+\sqrt{\nu \varepsilon}}
$$

where

$$
C_{1 \varepsilon}=\max \left[0.43, \frac{\eta}{\eta+5}\right]
$$

with

$$
\eta=S \frac{k}{\varepsilon}
$$

The default model constants are

$$
C_{2 \varepsilon}=1.9, \sigma_{k}=1.0, \sigma_{\varepsilon}=1.2
$$

Nevertheless, before the model is used to simulate rotor wakes, it must be calibrated for free stream conditions in order to ensure equilibrium inside the SBL of the inlet profiles with the ground and the turbulence model.

From equation (17), a value of $C_{\mu}$ can be evaluated for the wind speed gradients and mean strain tensor typical of the SBL and Monin-Obukhov theory.

The main component of the strain tensor at the SBL can be derived as

$$
S_{i j}=S_{12}=S_{21}=\frac{1}{2}\left(\frac{\partial U}{\partial z}\right)=\frac{1}{2}\left(\frac{u_{*}}{\kappa z}\right)
$$

From this result, the rest of analytical expressions (18) and (19) for the estimation of $A_{\mathrm{s}}$ parameter in the SBL remains:

$$
\begin{gathered}
\tilde{S}=\sqrt{S_{12} S_{21}+S_{21} S_{12}}=S_{12} \sqrt{2}, \quad S_{i j} S_{j k} S_{k i}=S_{12} S_{21} S_{12}+S_{21} S_{12} S_{21}=2 S_{12}^{3} \\
W=\frac{2 S_{12}^{3}}{\left(\sqrt{2} S_{12}\right)^{3}}=\frac{1}{\sqrt{2}}, \quad \phi=\left(\frac{1}{3}\right) \frac{1}{\cos \left(\frac{\sqrt{6}}{\sqrt{2}}\right)} \approx 0.333 \\
A_{s}=\sqrt{6} \cos (\phi)=\sqrt{6} \cos (0.333) \approx \sqrt{6}
\end{gathered}
$$

The resulting set of parameters (25) and (26) are then evaluated using equation (17) together with equation (24) and the $k$ and $\varepsilon$ profiles (3) and (4) for a value of $C_{\mu}=0.033$, resulting in a value of $C_{\mu}=0.064$. In the same way, the mean strain tensor (11) as well as the $k$ and $\varepsilon$ profiles (3) and (4) are also evaluated through equations (21) and (22), getting a value of $C_{1 \varepsilon}=0.52$.

These results agree with the simulation that is based on FLUENT 6.3 for a free stream flow in an empty domain at the inlet. Figure 3 shows the axial evolution at hub height of $C_{\mu}$ and $C_{1 \varepsilon}$ according to equations (17) and (21) when the $k$ and $\varepsilon$ profiles of the SBL are introduced at the inlet with a value of $C_{\mu}=0.033$. The results show, for this case, that these values are lost and that no equilibrium is reached along the domain as seen from the evolution of $C_{\mu}$ and $C_{1 \varepsilon}$, whereas a default value of $C_{\mu}=0.09$, if checked, leads indeed to equilibrium if it is used at the inlet for a similar simulation.

From this result, it is concluded that the realizable turbulence model, as defined in FLUENT 6.3, leads to equilibrium just for the default constants.

Because of this circumstance, the model has been tested using a free stream turbulence intensity associated with the value of $C_{\mu}=0.09$ (only in this simulation) in order to get equilibrium, instead of using the ABL characteristic value of $C_{\mu}=0.033$ that has been used in the rest of the paper. 


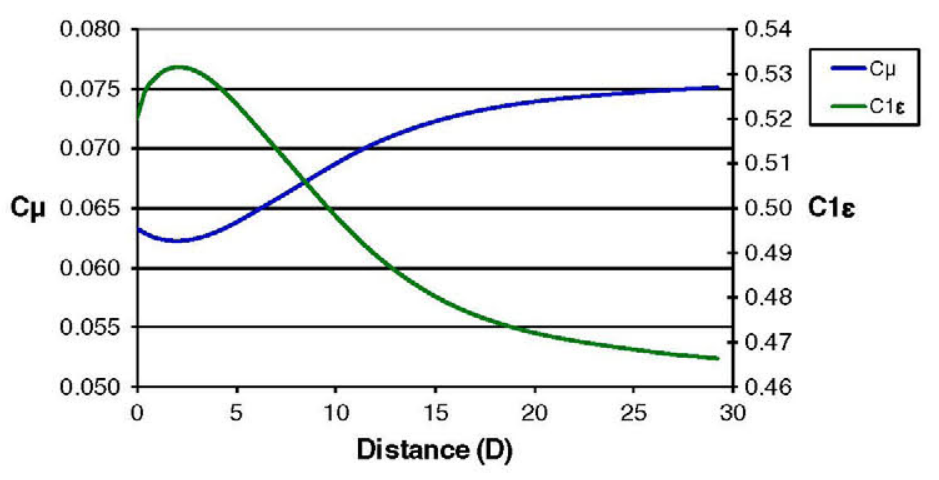

Figure 3. Axial evolution of $C_{\mu}$ and $C_{1 \varepsilon}$ along the domain under free stream conditions.

\subsection{Anisotropic Reynolds Stress Model (RSM)}

Another approach of simulating the turbulence mixing in the wake is to consider the anisotropic resolution of the fluid dynamics equations, by solving all the terms in the Reynolds stress tensor, through the introduction of seven extra equations into the system configuring thus the so-called RSM. Among the different versions existing in the literature of RSM, the model proposed by Gibson and Launder ${ }^{10}$ is used because it is the only model available in the solver FLUENT 6.3 that has proven accuracy for many flow cases.

The model takes into account all the effects derived from rapid changes at the main strain tensor in a more rigorous way than one or two equations of turbulence models. It has a great potential when carrying out simulations for complex flows, although it implies an increase in the computing time.

Nevertheless, the accuracy of RSM is still limited to the closure hypothesis used at the transport equations of the stress tensor, particularly those referred to the turbulent diffusion, pressure gradient and dissipation. The rest of the terms of the equations (convection, molecular diffusion and production) do not require to be specifically modeled.

Besides the seven equations prescribed for the Reynolds stress tensor, the RSM model, as implemented in FLUENT 6.3, solves the $k$ and $\varepsilon$ equations as well in a similar way to the standard $k-\varepsilon$ model. The value of $k$ obtained from this model is used for the definition of the Reynolds stress values at the wall-adjacent cells with the use of a local coordinate system. Defining $\tau$ as the tangential coordinate, $\eta$ as the normal coordinate and $\lambda$ as the binormal coordinate, the Reynolds stress values at the wall-adjacent cells are defined according to FLUENT $6.3^{18}$ as

$$
\frac{\overline{u_{\tau}^{\prime 2}}}{k}=1.098, \quad \frac{\overline{u_{\eta}^{\prime 2}}}{k}=0.247, \quad \frac{\overline{u_{\lambda}^{\prime 2}}}{k}=0.655, \quad-\frac{\overline{u_{\tau}^{\prime 2} u_{\eta}^{\prime 2}}}{k}=0.255
$$

This equation is really a set of boundary conditions for the Reynolds stresses at the wall, obtained by using the log law (2) and assuming equilibrium near the wall, neglecting convection and diffusion in the transport equations of the Reynolds stresses. ${ }^{17}$ To obtain the $k$ value at these cells, FLUENT 6.3 solves the transport equation (7), although this value is only used for the estimation of equation (27) at the wall-adjacent cells.

For the rest of the domain, turbulent kinetic energy $k$ is computed from the normal components of the Reynolds stress tensor:

$$
k=\frac{1}{2} \overline{u_{i}^{\prime} u_{i}^{\prime}}
$$

Both $k$ and $\varepsilon$ equations together with the continuity equation, the three momentum equations and the seven extra equations for solving the Reynolds stress tensor makes the RSM be composed of 13 equations to be solved.

The values of the Reynolds stress tensor components $\overline{u_{i}^{\prime} u_{j}^{\prime}}$ typical of the SBL proposed by Panofsky and Dutton ${ }^{13}$ are introduced at the inlet boundary, keeping for numerical stability the standard configuration as implemented in FLUENT 6.3. These values are defined according to FLUENT $6.3^{18}$ as

$$
\tau_{x z}=\rho u_{*}^{2} ; \quad \tau_{x x}=-2.4^{2} \rho u_{*}^{2} ; \quad \tau_{y y}=-1.92^{2} \rho u_{*}^{2} ; \tau_{z z}=-1.25^{2} \rho u_{*}^{2} ; \quad \tau_{x y}=\tau_{y x}=0
$$

Equation (29) can be reformulated in similar terms to those of equation (27) as

$$
\frac{\overline{u_{x}^{\prime 2}}}{k}=1.05, \quad \frac{\overline{u_{y}^{\prime 2}}}{k}=0.29, \quad \frac{\overline{u_{z}^{\prime 2}}}{k}=0.67, \quad-\frac{\overline{u_{x}^{\prime 2} u_{y}^{\prime 2}}}{k}=0.18
$$


As can be observed, the difference between both sets of shear stresses, at the wall (equation (27)) and at the entrance (equation (30)), is small so that the influence on the results at hub height is not expected to be important.

\section{RESULTS}

Results are structured in three parts, in terms of wind speed deficit, added turbulence intensity and shear stress $\left\langle u^{\prime} v^{\prime}\right\rangle$ at $2.5 D, 5.5 D$ and $8 D$ downstream of the wind turbine.

In order to complement the results, a comparison of the elliptic wake model with other existing wake models, such as the parabolic model UPMPARK, the anisotropic model UPMANIWAKE, and a wake model based similarly on the actuator disk technique but solved from an LES model, is included.

UPMPARK, proposed by Crespo et al. ${ }^{24}$ solves through a finite difference method the parabolic equations of fluid dynamics, neglecting thus the turbulent diffusion and pressure term in the main flow direction. A $k-\varepsilon$ turbulent closure is also used for the solution of turbulent transport. Unlike previous elliptic models, forces are not prescribed at the rotor disk. Instead, the velocity deficit is imposed at a section located $2.25 \mathrm{D}$ downstream of the wind turbine, where the wake is already expanded. ${ }^{19,20}$

On the other hand, UPMANIWAKE, proposed by Gómez-Elvira et al.,$^{21}$ is based on an explicit algebraic model for the solution of the Reynolds stress tensor, neglecting the diffusion and convection terms at the conservation equations and matching the production and dissipation terms, which allows solving the equations in an algebraic manner.

Similarly, these models are compared with another one based on LES for solving the atmospheric flow as well as the wake area, as presented by Jimenez et al. ${ }^{22}$ The wind turbine is also simulated as an actuator disk over which drag forces are applied at the grid points coincident with the rotor.

Because of data availability, only UPMPARK is included at the comparison with the elliptic model, for both wind speed deficit and added turbulence intensity.

As indicated in Section 1, the focus of this paper is on the comparison of elliptic models with experiments; nevertheless, it may be of interest for engineering applications to also compare with parabolic models that, although are less accurate, consume less computing time.

\subsection{Wind speed deficit}

Figures 4 describe the lateral evolution of the wind speed deficit for the different turbulent schemes as a function of the incoming wind direction compared with the experimental data at each mast. Wind direction refers to relative direction from the incoming flow, so that $0^{\circ}$ indicates the center of the rotor, where the maximum wind speed deficit is expected.

The main and primary result of the analysis leads to the underestimation of the wind speed deficit by the standard form of the $k-\varepsilon$ turbulence model. This result, mainly due to the excess of turbulence diffusion in the wake, makes the flow recover much earlier than expected, leading to values of more than $90 \%$ in the far wake section, as observed in Figure 4 .

The proposed corrections on the $k-\varepsilon$ standard default model show a better agreement with the measurements, especially at the $5.5 D$ and $8 D$ sections.

The local increase in the turbulent dissipation rate makes the turbulence mixing be mitigated so that the recovery of the flow is slower, obtaining more realistic wind speed deficits.

The anisotropic treatment of the flow through the RSM model gets also very acceptable results not only in the far wake but also in the near wake, and a more accurate prediction of the wake expansion downstream of the wind turbine.
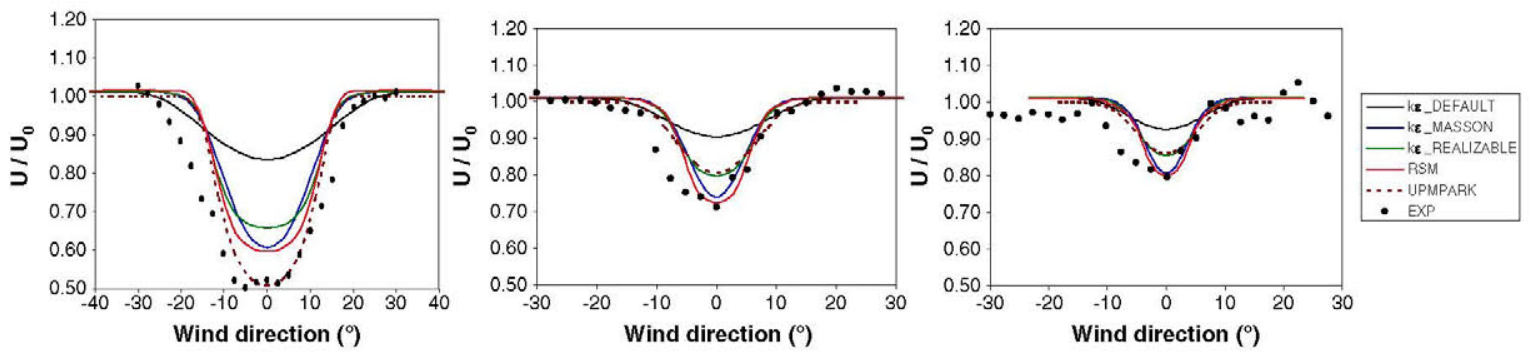

Figure 4. Wind speed deficit in the flow direction at a downstream distance of $2.5 D$ (left), $5.5 D$ (center) and $8 D$ (right). Comparison of $k-\varepsilon$ default ${ }^{8} k-\varepsilon$ Masson, ${ }^{7} k-\varepsilon$ realizable, ${ }^{9}$ Reynolds stress model (RSM) ${ }^{10}$ and UPMPARK. ${ }^{24}$ 
A better agreement is observed for UPMPARK not only for the estimation of the maximum wind speed deficit but also for the estimation of the wake expansion. The elliptic approximations remain too diffusive at this section located in the near wake.

However, the results in the far wake are more accurate for the elliptic models, in particular, for the corrections on the $k-\varepsilon$ model proposed by Masson as well as for the anisotropic model RSM. In the far wake, the parabolic model underestimates the wind speed deficit in comparison with the previous modifications on the elliptic models.

Both type of models predict a similar wake expansion at the different sections inside the wake, except for the RSM model that estimates a wider wake both at the near wake and far wake sections.

\subsection{Added turbulence intensity}

The derived results from the $k-\varepsilon$ turbulence model for the estimation of the total turbulence intensity assumes the hypothesis of isotropy in the added turbulence and anisotropy in the free stream. This makes total turbulence intensity in the wake to be computed according to Schepers ${ }^{23}$ as

$$
T I_{\text {total }}=\frac{1}{U_{0}} \sqrt{0.666 \cdot k_{\text {total }}+0.38027 \cdot k_{\text {freestream }}}
$$

The turbulence intensity derived for the RSM model is directly prescribed from the variance of the axial wind speed component.

In order to normalize the results with respect to the free stream conditions, the added turbulence intensity is estimated from the free stream and the total turbulence intensity in the wake as

$$
T I_{\text {added }}=\sqrt{T I_{\text {total }}^{2}-T I_{\text {freestream }}^{2}}
$$

The results in Figure 5 show that, although the RANS elliptic model underestimates the added turbulence intensity in the near wake, the prediction is very acceptable at the sections located in the far wake, especially in the area near the wake axis.

Local corrections on the prognostic equation for $\varepsilon$ as well as for the anisotropic RSM make the high shear zones be merged into the wake axis at a section further than predicted by the standard form of the $k-\varepsilon$ turbulence model, as observed from the measurements.

The modeling of turbulence intensity through the parabolic model UPMPARK as well as through the UPMANIWAKE model and the LES-based model offer outstanding results in the near wake, especially for the estimation of the shear layers at both sides of the wake axis (Figure 4). The elliptic model leads to an underestimation on the turbulence intensity at both shear layers.

Nevertheless, the estimation of the added turbulence intensity made by UPMANIWAKE fails at the far wake section, and UPMPARK overestimates its value at the center of the wake with a single high shear stress zone. The LES model as well as the modified elliptic models give an accurate representation of the turbulence intensity at the far wake, particularly at its central axis.
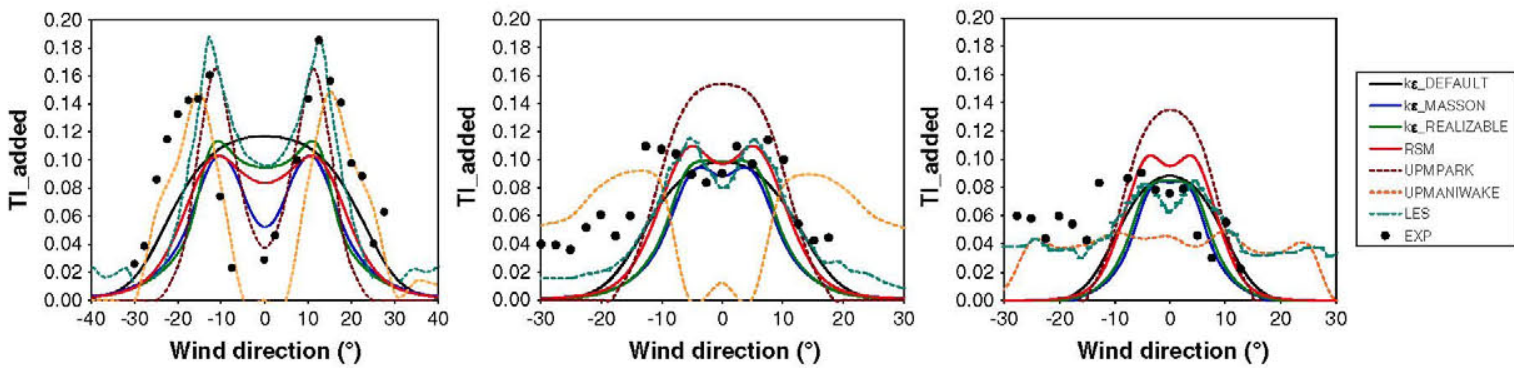

Figure 5. Added turbulence intensity in the average flow direction at a downstream distance of $2.5 D, 5.5 D$ and $8 D$. Comparison of $k-\varepsilon$ default, ${ }^{8} k-\varepsilon$ Masson, ${ }^{7} k-\varepsilon$ realizable, ${ }^{9}$ Reynolds stress model (RSM), ${ }^{10}$ UPMPARK ${ }^{24}$ UPMANIWAKE, ${ }^{21}$ and large eddy simulation (LES). ${ }^{22}$ 


\section{3. $\left\langle u^{\prime} v^{\prime}>\right.$ shear stress}

The Boussinesq assumption allows reproducing shear stress for the isotropic models from the turbulent viscosity concept and the mean velocity gradients. At the horizontal plane, shear stress $\left\langle u^{\prime} v^{\prime}\right\rangle$ can be obtained from the corresponding relationship:

$$
<u^{\prime} v^{\prime}>=-\mu_{\mathrm{t}}\left(\frac{\partial u}{\partial y}+\frac{\partial v}{\partial x}\right) \approx-\mu_{\mathrm{t}} \frac{\partial u}{\partial y}
$$

Where assuming $X$-axis as the main flow direction and $Y$-axis the cross direction, the value of $\left\langle u^{\prime} v^{\prime}>\right.$ shear stress comes almost entirely from the cross variation of the axial velocity component.

Figure 6 shows the lateral evolution of $\left\langle u^{\prime} v^{\prime}\right\rangle$ shear stress at the downstream sections, where results for the UPMANIWAKE and LES models are only available for the near section at $2.5 \mathrm{D}$.

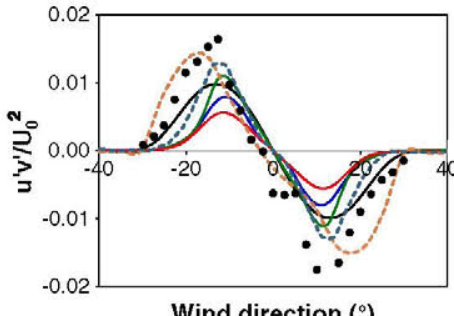

Wind direction $\left({ }^{\circ}\right)$

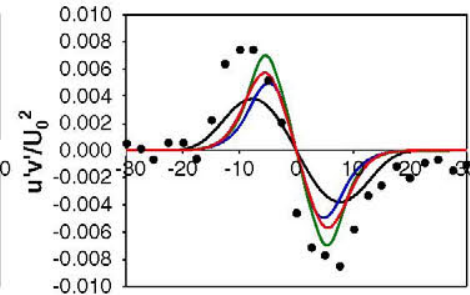

Wind direction $\left({ }^{\circ}\right)$

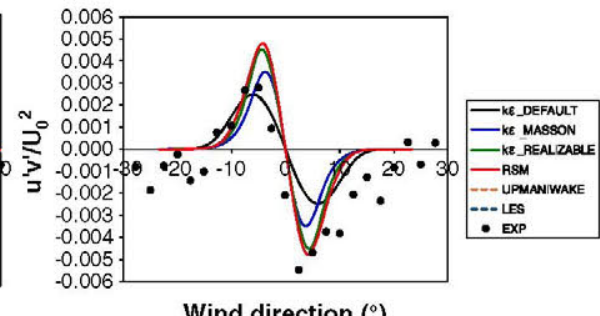

Wind direction $\left({ }^{\circ}\right)$

Figure 6. Shear stress $\left\langle u^{\prime} v^{\prime}\right\rangle$ in the average flow direction at a downstream distance of 2.5D, 5.5D and 8D. Comparison of $k-\varepsilon$ default, ${ }^{8} k-\varepsilon$ Masson, ${ }^{7} k-\varepsilon$ realizable, ${ }^{9}$ Reynolds stress model (RSM), ${ }^{10}$ UPMANIWAKE, ${ }^{21}$ and large eddy simulation (LES). ${ }^{22}$

The comparison of modeled shear stress against experimental data at $2.5 \mathrm{D}$ downstream lead to an underestimation of the maximum shear stress values at the area between the wake and free stream fluid zones, located at approximately $\pm 12^{\circ}$. Despite the improvement at the definition of the turbulent viscosity at that area, wind speed gradients are not so intensive as expected. The anisotropic model RSM cannot reproduce at these sections the expected shear stress level at the wake border, leading to lower values, than the isotropic models.

UPMANIWAKE and LES models estimate values of the shear stress closer to the experimental measurements at the areas of strong gradients, leading to deeper and wider wakes, as observed also in Figure 6 from the added turbulence intensity results. On the other hand, the results obtained at both sides of the wake axis are similar to the rest of the models.

Nevertheless, the modeling results are improved at the far wake, except for the standard form of the $k-\varepsilon$ turbulence model that continues underestimating $\left\langle u^{\prime} v^{\prime}\right\rangle$ shear stress at those positions. The modification on the turbulent viscosity for the realizable $k-\varepsilon$ and on the $\varepsilon$ equation for the Masson's proposal lead to a significant improvement at sections $5.5 D$ and $8 D$ downstream, where the anisotropic RSM model shows a better agreement as well.

\section{CONCLUSIONS}

The standard form of the $k-\varepsilon$ turbulence model for the simulation of single rotor wakes in the SBL leads to an underestimation of the wind speed deficit. Local corrections on the $\varepsilon$ equation should be made in order to counteract the excess of turbulent diffusion in the near wake flow produced by the standard $k-\varepsilon$ turbulence model. Some of them give very acceptable results in the far wake region where wind turbines are usually placed. Higher-order turbulence models such as the anisotropic RSM look quite promising and offer also accurate predictions of the wind speed deficit in the far wake. This anisotropic model offer an interesting and economic alternative to the isotropic $k-\varepsilon$ turbulence models on the basis of the eddy viscosity concept, unable, so far, to solve accurately the imbalance on the turbulent length scales between the free stream and the wake area. Some of the proposed modifications on the standard form of the $k-\varepsilon$ model offer, in general, very outstanding results in the far wake sections, but they are still very dependent on how their parameters are tuned for different cases.

The simulation of $\left\langle u^{\prime} v^{\prime}>\right.$ shear stress, estimated from the Boussinesq assumption, offer accurate results especially at $5.5 D$ and $8 D$ downstream of the wind turbine.

The use of wake models based on parabolic approximations such as UPMPARK or on other anisotropic models such as UPMANIWAKE allows improving significantly the results in the near wake with respect to the elliptic models if no corrections are made on them. However, the results improve in the far wake for some proposed modifications on the $\varepsilon$ equation of the elliptic models (in particular, Masson's correction) and the RSM model, allowing to estimate accurately the maximum 
wind speed deficit and the turbulence intensity at the center of the wake. On the other hand, parabolic models require less computing resources, and it may be worthwhile to investigate if parabolic models with modified closure schemes will give better results also in the far wake.

Described RANS models combined with the actuator disk technique based on linear momentum theory lead to acceptable predictions, but they will never be as accurate as space filtering models such as LES models for the prediction of the near wake flow. That is why these more economic models based on lower-order turbulent closures may be very feasible for wind farm analysis applications where the main interest relies on the estimation of the far wake flow, whereas LES modeling may be more interesting for local studies of rotor aerodynamics, with a significant increase on the computational effort, not affordable for an operational use.

The results derived from this analysis and the proposed modifications on the standard form of the $k-\varepsilon$ turbulence models correspond to a stand-alone wind turbine, but no conclusion is reached on how these schemes would behave in wind farm environments and under other atmospheric conditions. On the other hand, more research will be needed on how this turbulence modeling remains accurate on modern wind turbines located at more than $100 \mathrm{~m}$ high. The present state of the art suggests that rotor modeling could remain valid; however, the modeling of the turbulence should be revised by using advanced wind flow models that could allow solving properly the wide range of turbulent length scales present in the ABL including areas of big wind farms with modern wind turbines.

With that purpose, more validations are needed from wind farm measurements in order to observe how different microscale effects such as wake-terrain interaction or wakes merging at large complex terrain wind farms or thermal effects derived from atmospheric stability (especially at offshore conditions) or the different turbulent length scales can influence the evolution of wakes.

Similarly, different aspects such as mesh refinement around the shear layer of the wake or grid control at different inflow wind directions will also be analyzed in order to optimize wind farm models in the short term.

\section{REFERENCES}

1. Vermeer LJ, et. al. Wind turbine wake aerodynamics. Progress in Aerospace Sciences 2003; 39: 467-510.

2. Crespo A, Hernández J, Frandsen S. A survey of modelling methods for wind-turbine wakes and wind farms. Wind Energy 1999; 2: 1-24.

3. Barthelmie RJ, Frandsen ST, Rathmann O, Politis E, Prospathopoulos J, Rados K, Hansen K, Cabezón D, Schlez W, Phillips J, Neubert A, van der Pijl S, Schepers G. Flow and wakes in large wind farms in complex terrain and offshore, European Wind Energy Conference, Brussels, 2008. (Scientific track).

4. Réthoré P, Sørensen N, Zahle F, Bechmann A. Study of the wake turbulence of a CFD actuator disk model compared with a full rotor CFD model, Proceedings of the European Wind Energy Conference, 2009. Marseille (France).

5. Cabezón D, Sanz J, Martí I, Crespo A. CFD modelling of the interaction between the surface boundary layer and rotor wake. Comparison of results obtained with different turbulence models and mesh strategies, European Wind Energy Conference and Exhibition 2009, Marseille, 2009.

6. Prospathopoulos, et al. Evaluation of the effects of turbulence model enhancements on wind turbine wake predictions. Journal Wind Energy 2011; 14: 285-300.

7. El Kasmin A, Masson C. An extended k- $\varepsilon$ model for turbulent flow through horizontal-axis wind turbines. Journal of Wind Engineering and Industrial Aerodynamics 2008; 96: 103-122.

8. Launder BE, Spalding DB. Lectures in Mathematical Models of Turbulence. Academic Press: London, England, 1972.

9. Shih $\mathrm{T}$, Liou W, et. al. A new k-e eddy viscosity model for high Reynolds number turbulent flows. Journal of Computers Fluids 1995; 24: 227-238.

10. Gibson MM, Launder BE. Ground effects on pressure fluctuations in the atmospheric boundary layer. Journal of Fluid Mechanics 1978; 86: 491-511.

11. Cleijne JW. Results of Sexbierum wind farm. Report MT-TNO Apeldoorn, 1992.92-388.

12. Sanz Rodrigo J, Cabezón D, Martí I, Patilla P, van Beeck J. Numerical CFD modelling of non-neutral atmospheric boundary layers for offshore wind resource assessment based on Monin-Obukhov theory, EWEC 2008 scientific proceedings, Brussels, Belgium, 2008.

13. Panofsky H, Dutton J. Atmospheric Turbulence. Wiley: New York, 1984.

14. Burton T, Sharpe D, et al. Wind Energy Handbook. John Wiley \& Sons LTD: England, 2001.

15. Blocken B, Stathopoulos T, Carmeliet J. CFD simulation of the atmospheric boundary layer - wall function problems. Joumal Atmospheric Environment. 
16. Barth TJ, Jespersen D. The design and application of upwind schemes on unstructured meshes. AIAA paper 89-0366, 1989.

17. Richards PJ, Hoxey RP. Appropiate boundary conditions for computational wind engineering models using the K-EPS turbulence model. Journal of Wind Engineering and Industrial Aerodynamics 1993; 46-47: 145-153.

18. ANSYS FLUENT 6.3 User's Guide, 2009.

19. Crespo A, Hernández J. Numerical modelling of the flow field in a wind turbine wake, Proceedings of the 3rd Joint ASCE/ASME Mechanics Conference, Forum on Turbulent Flows. ASME, FED-vol. 76, La Jolla, CA, USA, 1989; 121-7.

20. Crespo A, Manuel F, Hernández J. Numerical modelling of wind turbine wakes, European Community Wind Energy Conference, Proceedings, Madrid, 1990.

21. Gómez-Elvira R, et al. Anisotropiy of turbulence in wind turbine wakes. Journal of Wind Engineering and Industrial Aerodynamics 2003; 93: 797-814.

22. Jimenez A, Crespo A, et al. Large-eddy simulation of a wind turbine wake, Proceedings of the European Academy of Wind Energy Seminar, Pamplona (Spain), 2007.

23. Schepers JG. ENDOW: Validation and Improvement of ECN's Wake Model. ECN:ECN-C-03-034: Petten: The Netherlands, 2003. 113.

24. Crespo A, Chacón L, Hernández J, Manuel F, Grau JC. UPMPARK: a parabolic 3D code to model wind farms, Proceedings of EWEC '94, Thessaloniki, Greece, 1994; 454-9. 